\title{
Idler-Resonant Femtosecond Optical Parametric Oscillator with High Mid-Infra-Red Beam Quality
}

\author{
Lin Xu, David P. Shepherd, David J. Richardson and Jonathan H.V. Price \\ Optoelectronics Research Centre, University of Southampton, Southampton SO17 1BJ, UK tel: +44 23 8059 3144; fax: +44 2380593142 \\ l.xu@soton.ac.uk
}

\begin{abstract}
We report an idler-resonant femtosecond optical parametric oscillator (OPO) with average output power of $520 \mathrm{~mW}$, repetition-rate of $80 \mathrm{MHz}$, pulse duration of $90 \mathrm{fs}$ and nearly diffraction-limited beam quality at $\sim 2.4 \mu \mathrm{m}$.

OCIS codes: (140.3460) Lasers; (190.4970) Parametric oscillators and amplifiers
\end{abstract}

\section{Introduction}

Femtosecond laser sources in the mid-infrared (mid-IR) range have found an increasing number of applications in the fields of spectroscopy, metrology, and biology. High peak-power femtosecond pump lasers have been used to generate broad-band supercontinuum in highly nonlinear fiber covering a wide spectral range between the visible and mid-IR[1]. Non-silica fiber has the potential to produce continuum spectra in the 3.0-5.0 $\mu$ m wavelength range widely used for spectroscopic applications [2, 3], but this requires diffraction-limited beam quality and, for high coherence continua, femtosecond pump pulses at wavelengths in the 2.0-2.5 $\mu \mathrm{m}$ region. Fiber-laser-pumped OPOs are recognised as promising pump laser candidates for this application and in this paper we present an unusual combination of an idler-resonant OPO, synchronously pumped by a femtosecond Yb:fiber MOPA system in a ring cavity, producing $90 \mathrm{fs}$ idler pulses with nearly diffraction-limited beam quality. The average output power was as high $520 \mathrm{~mW}(6.5 \mathrm{~nJ}$ pulse energy) at a wavelength of $2.4 \mu \mathrm{m}$.

\section{System setup and experimental results}

The schematic of the femtosecond OPO is shown in Fig. 1. The pump source is an $80 \mathrm{MHz}$ Yb:fiber MOPA system operating with a linear polarization and beam quality of $\mathrm{M}^{2}=1.05$. The central wavelength is $1055 \mathrm{~nm}$ with average power of $4.5 \mathrm{~W}$ and pulse duration of $130 \mathrm{fs}$ (full details will be reported elsewhere). The pump beam is focused into a periodically poled $5 \% \mathrm{MgO}$-doped $\mathrm{LiNbO}_{3}$ (PPLN) crystal with a $1 / \mathrm{e}^{2}$ intensity beam waist radius of $75 \mu \mathrm{m}$. The $1 \mathrm{~mm}$-long PPLN crystal (MOPO1-1.0-1 from Covesion Ltd.) is mounted inside an oven to allow temperature tuning and contains five gratings with periods ranging from 29.5 to $31.5 \mu \mathrm{m}$ in steps of $0.5 \mu \mathrm{m}$. In this experiment, only the $31.5-\mu \mathrm{m}$ grating period is used as this was matched to the idler wavelength range covered by our HR mirror coatings. The anti-reflection coating on the commercially available PPLN crystal was optimised for signal wavelengths and had a reflectivity of approximately $\sim 3.5 \%$ at the idler wavelengths generated here. We therefore chose to operate the OPO in a ring-geometry, rather than using a standing-wave cavity, to minimise the effect of the losses on the power conversion efficiency. The OPO cavity comprised four plane and two concave spherical mirrors. The curved mirrors have a radius of curvature of $300 \mathrm{~mm}$ and all six mirrors are coated for high reflectivity (HR) across the 2200 2700 nm range. The plane mirror M6 is set on a piezo translation stage to fine tune the cavity length. A pellicle beam splitter inserted between M5 and M6 acted as an output coupler and the reflectivity could be angletuned from approximately $28 \% \sim 46 \%$ at the idler wavelength.

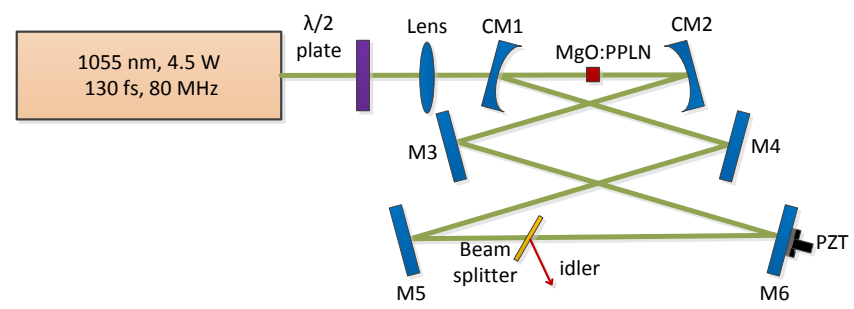

Fig. 1. Experimental setup of the OPO

Fig. 2 shows the signal and idler spectra at a crystal temperature of $100{ }^{\circ} \mathrm{C}$, with tuning achieved by adjusting the cavity length. The OPO signal and corresponding idler wavelengths can be tuned from $1850 \sim 1970 \mathrm{~nm}$ and $2250 \sim 2450 \mathrm{~nm}$, respectively. A maximum average idler power of $520 \mathrm{~mW}$ is obtained at a wavelength of $2440 \mathrm{~nm}$, 
corresponding to a conversion efficiency of $11.5 \%$. Fig. 3 shows the output power vs. pump power, indicating a pump threshold of $2.2 \mathrm{~W}$ and slope efficiency of $23 \%$. Tuning across the full reflectivity range of the mirrors is being investigated and may require purging of the system to avoid atmospheric absorption.

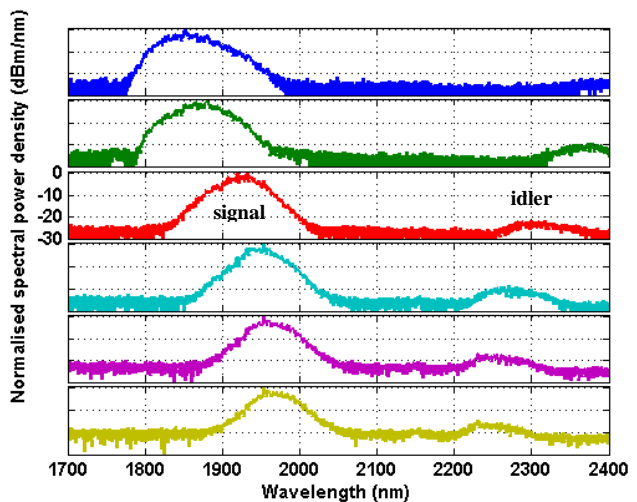

Fig. 2. Signal and idler spectra tuning achieved by changing the cavity length. (Data range limited by spectrum analyser Yokogawa AQ6375)

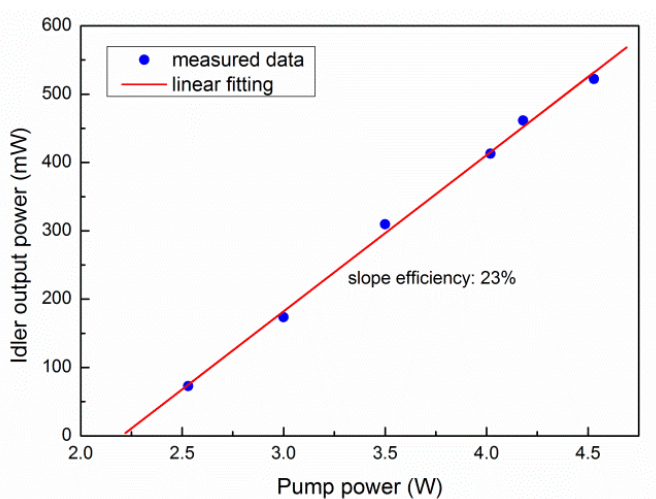

Fig. 3. Idler output power vs. launched pump power.

With the idler centered at $2.44 \mu \mathrm{m}$, the autocorrelation trace (Fig. 4) had a full-width-half-maximum duration of $118 \mathrm{fs}$, which gives a pulse duration of $90 \mathrm{fs}$ when using a Gaussian pulse shape with best-fit to the data. The idler pulse is therefore somewhat shorter than the pump pulse, which may be due to the group velocity mismatch of the pump and oscillating waves in the nonlinear crystal [4]. Fig. 5 shows a beam quality measurement of the idler at the maximum power, with $\mathrm{M}_{\mathrm{x}}{ }^{2}=1.07$ and $\mathrm{M}_{\mathrm{y}}{ }^{2}=1.03$ measured in the horizontal and vertical planes, respectively.

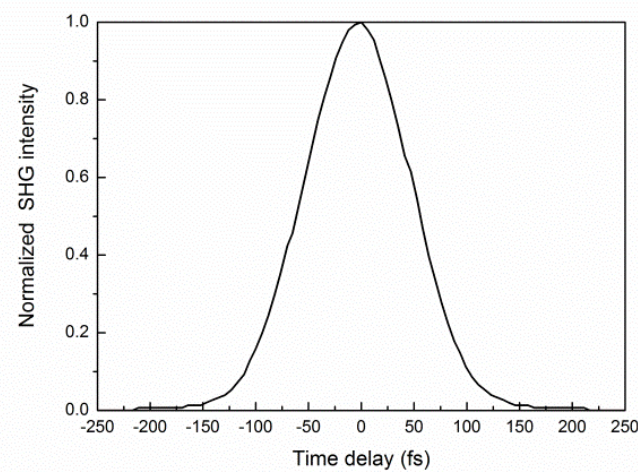

Fig. 4. Autocorrelation of idler wave.

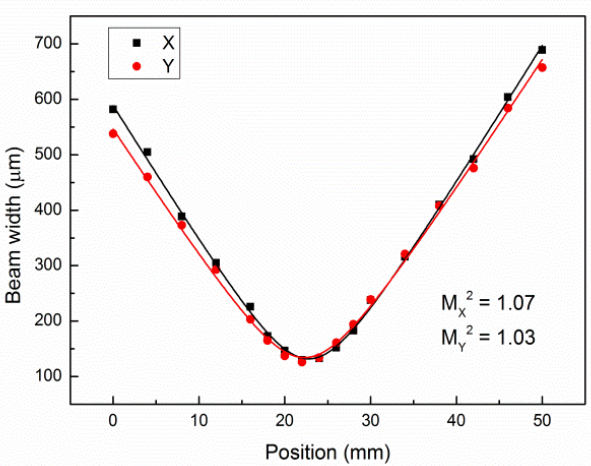

Fig. 5. $\mathrm{M}^{2}$ measurement in horizontal and vertical planes

\section{Conclusion}

We have demonstrated an $80 \mathrm{MHz}$ Yb:fiber MOPA pumped, idler-resonant, synchronously pumped femtosecond OPO producing up to $520 \mathrm{~mW}$ of average power, pulse durations of $90 \mathrm{fs}$ and wavelength tunable from $2250-2450 \mathrm{~nm}$. These operating parameters and the nearly diffraction-limited beam quality make this a promising source for pumping soft-glass fibers for supercontinuum generation further into the mid-IR e.g. for spectroscopy applications.

This work was undertaken with funding from EPSRC UK (EP/H049371/1 and EP/I02798X/1). Florian Emaury (ETH, Zurich), James Feehan, Ho Yin Chan and Simon Teh provided technical assistance with the pump source.

\section{References}

[1] P. Domachuk, N. A. Wolchover, M. Cronin-Golomb, A. Wang, A. K. George, C. M. B. Cordeiro, J. C. Knight, and F. G. Omenetto, "Over $4000 \mathrm{~nm}$ bandwidth of mid-IR supercontinuum generation in sub-centimeter segments of highly nonlinear tellurite PCFs," Optics Express 16, 7161-7168 (2008).

[2] J. H. V. Price, T. M. Monro, H. Ebendorff-Heidepriem, F. Poletti, P. Horak, V. Finazzi, J. Y. Y. Leong, P. Petropoulos, J. C. Flanagan, G. Brambilla, F. Xian, and D. J. Richardson, "Mid-IR Supercontinuum Generation From Nonsilica Microstructured Optical Fibers," IEEE J. Sel. Topics Quantum Electron. 13, 738-749 (2007).

[3] J. H. V. Price, X. Feng, A. M. Heidt, G. Brambilla, P. Horak, F. Poletti, G. Ponzo, P. Petropoulos, M. Petrovich, J. Shi, M. Ibsen, W. H. Loh, H. N. Rutt, and D. J. Richardson, "Supercontinuum generation in non-silica fibers," Optical Fiber Technology 18, 327-344 (2012).

[4] J. D. V. Khaydarov, J. H. Andrews, and K. D. Singer, "Pulse compression in a synchronously pumped optical parametric oscillator from group-velocity mismatch," Optics Letters 19, 831-833 (1994). 